\title{
Strengthened Brooks' Theorem for digraphs of girth at least three
}

\author{
Ararat Harutyunyan* \\ Department of Mathematics \\ Simon Fraser University \\ Burnaby, B.C. V5A 1S6, Canada \\ aha43@sfu.ca
}

\author{
Bojan Mohar ${ }^{\dagger \ddagger}$ \\ Department of Mathematics \\ Simon Fraser University \\ Burnaby, B.C. V5A 1S6, Canada \\ mohar@sfu.ca
}

Submitted: Dec 30, 2010; Accepted: Sep 16, 2011; Published: Oct 3, 2011

Mathematics Subject Classification: 05C15, 05C20

\begin{abstract}
Brooks' Theorem states that a connected graph $G$ of maximum degree $\Delta$ has chromatic number at most $\Delta$, unless $G$ is an odd cycle or a complete graph. A result of Johansson shows that if $G$ is triangle-free, then the chromatic number drops to $O(\Delta / \log \Delta)$. In this paper, we derive a weak analog for the chromatic number of digraphs. We show that every (loopless) digraph $D$ without directed cycles of length two has chromatic number $\chi(D) \leq\left(1-e^{-13}\right) \tilde{\Delta}$, where $\tilde{\Delta}$ is the maximum geometric mean of the out-degree and in-degree of a vertex in $D$, when $\tilde{\Delta}$ is sufficiently large. As a corollary it is proved that there exists an absolute constant $\alpha<1$ such that $\chi(D) \leq \alpha(\tilde{\Delta}+1)$ for every $\tilde{\Delta}>2$.
\end{abstract}

Keywords: Digraph coloring, dichromatic number, Brooks theorem, digon, sparse digraph.

\section{Introduction}

Brooks' Theorem states that if $G$ is a connected graph with maximum degree $\Delta$, then $\chi(G) \leq \Delta+1$, where equality is attained only for odd cycles and complete graphs. The presence of triangles has significant influence on the chromatic number of a graph. A

*Research supported by FQRNT (Le Fonds québécois de la recherche sur la nature et les technologies) doctoral scholarship.

†Supported in part by an NSERC Discovery Grant (Canada), by the Canada Research Chair program, and by the Research Grant P1-0297 of ARRS (Slovenia).

$\ddagger$ On leave from: IMFM \& FMF, Department of Mathematics, University of Ljubljana, Ljubljana, Slovenia. 
result of Johansson [7] states that if $G$ is triangle-free, then $\chi(G)=O(\Delta / \log \Delta)$. In this note, we study the chromatic number of digraphs [4], [9], [12] and show that Brooks' Theorem for digraphs can also be improved when we forbid directed cycles of length 2 .

\section{Digraph colorings and the Brooks Theorem}

Let $D$ be a (loopless) digraph. A vertex set $A \subset V(D)$ is called acyclic if the induced subdigraph $D[A]$ has no directed cycles. A $k$-coloring of $D$ is a partition of $V(D)$ into $k$ acyclic sets. The minimum integer $k$ for which there exists a $k$-coloring of $D$ is the chromatic number $\chi(D)$ of the digraph $\mathrm{D}$. The above definition of the chromatic number of a digraph was first introduced by Neumann-Lara [12]. The same notion was independently introduced much later by the second author when considering the circular chromatic number of weighted (directed or undirected) graphs [9]. The chromatic number of digraphs was further investigated by Bokal et al. [4]. The notion of chromatic number of a digraph shares many properties with the notion of the chromatic number of undirected graphs. Note that if $G$ is an undirected graph, and $D$ is the digraph obtained from $G$ by replacing each edge with the pair of oppositely directed arcs joining the same pair of vertices, then $\chi(D)=\chi(G)$ since any two adjacent vertices in $D$ induce a directed cycle of length two. Another useful observation is that a $k$-coloring of a graph $G$ is a $k$-coloring of a digraph $D$, where $D$ is a digraph obtained from assigning arbitrary orientations to the edges of $G$. Mohar [10] provides some further evidence for the close relationship between the chromatic number of a digraph and the usual chromatic number. For digraphs, a version of Brooks' theorem was proved in [10]. Note that a digraph $D$ is $k$-critical if $\chi(D)=k$, and $\chi(H)<k$ for every proper subdigraph $H$ of $D$.

Theorem 1.1 ([10]). Suppose that $D$ is a $k$-critical digraph in which for every vertex $v \in V(D), d^{+}(v)=d^{-}(v)=k-1$. Then one of the following cases occurs:

1. $k=2$ and $D$ is a directed cycle of length $n \geq 2$.

2. $k=3$ and $D$ is a bidirected cycle of odd length $n \geq 3$.

3. $D$ is bidirected complete graph of order $k \geq 4$.

Neumann-Lara [12] first proved a tight upper bound on the chromatic number of a digraph.

Theorem 1.2 ([12]). Let $D$ be a digraph and denote by $\Delta_{o}$ and $\Delta_{i}$ the maximum outdegree and in-degree of $D$, respectively. Then

$$
\chi(D) \leq \min \left\{\Delta_{o}, \Delta_{i}\right\}+1 .
$$

In this paper, we study the chromatic number of digon-free digraphs. For digonfree planar digraphs, it was conjectured by Neumann-Lara [13], and independently, by Škrekovski in [4], that the chromatic number is at most 2. This conjecture is also related to a recent work of Aharoni, Berger and Kfir [1]. 
We study improvements of Theorem 1.2 for digon-free digraphs using the following substitute for the maximum degree. If $D$ is a digraph, we let

$$
\tilde{\Delta}=\tilde{\Delta}(D)=\max \left\{\sqrt{d^{+}(v) d^{-}(v)} \mid v \in V(D)\right\}
$$

be the maximum geometric mean of the in-degree and out-degree of the vertices. Observe that $\tilde{\Delta} \leq \frac{1}{2}\left(\Delta_{o}+\Delta_{i}\right)$, by the arithmetic-geometric mean inequality (where $\Delta_{o}$ and $\Delta_{i}$ are as in Theorem 1.2). We show that when $\tilde{\Delta}$ is large (roughly $\tilde{\Delta} \geq 10^{10}$ ), then every digraph $D$ without digons has $\chi(D) \leq \alpha \tilde{\Delta}$, for some absolute constant $\alpha<1$. We do not make an attempt to optimize $\alpha$, but show that $\alpha=1-e^{-13}$ suffices. To improve the value of $\alpha$ significantly, a new approach may be required.

It may be true that the following analog of Johansson's result holds for digon-free digraphs, as conjectured by McDiarmid and Mohar [8].

Conjecture 1.3. Every digraph $D$ without digons has $\chi(D)=O\left(\frac{\tilde{\Delta}}{\log \tilde{\Delta}}\right)$.

If true, this result would be asymptotically best possible in view of the chromatic number of random tournaments of order $n$, whose chromatic number is $\Omega\left(\frac{n}{\log n}\right)$ and $\tilde{\Delta}>$ $\left(\frac{1}{2}-o(1)\right) n$, as shown by Erdős et al. [5].

We also believe that the following conjecture of Reed generalizes to digraphs without digons.

Conjecture 1.4 ([14]). Let $\Delta$ be the maximum degree of (an undirected) graph $G$, and let $\omega$ be the size of the largest clique. Then

$$
\chi(G) \leq\left\lceil\frac{\Delta+1+\omega}{2}\right\rceil .
$$

If we define $\omega=1$ for digraphs without digons, we can pose the following conjecture for digraphs.

Conjecture 1.5. Let $D$ be a digraph without digons. Suppose that for every $v \in V(D)$, $d^{+}(v)=d^{-}(v)=\Delta$. Then

$$
\chi(D) \leq\left\lceil\frac{\Delta}{2}\right\rceil+1 .
$$

Conjecture 1.5 is trivial for $\Delta=1$, and follows from Lemma 3.2 for $\Delta=2,3$. We believe that the conjecture is also true for a general digon-free digraph with $\Delta$ replaced by $\tilde{\Delta}$.

\section{Basic definitions and notation}

We end this section by introducing some terminology that we will be using throughout the paper. The notation is standard and we refer the reader to [3] for an extensive treatment of digraphs. All digraphs in this paper are simple, i.e. there are no loops or multiple arcs in the same direction. We use $x y$ to denote the arc joining vertices $x$ and $y$, where $x$ is 
the initial vertex and $y$ is the terminal vertex of the arc $x y$. We denote by $A(D)$ the set of arcs of the digraph $D$. For $v \in V(D)$ and $e \in A(D)$, we denote by $D-v$ and $D-e$ the subdigraph of $D$ obtained by deleting $v$ and the subdigraph obtained by removing $e$, respectively. We let $d_{D}^{+}(v)$ and $d_{D}^{-}(v)$ denote the out-degree (the number of arcs whose initial vertex is $v$ ) and the in-degree (the number of arcs whose terminal vertex is $v$ ) of $v$ in $D$, respectively. The subscript $D$ may be omitted if it is clear from the context. A vertex $v$ is said to be Eulerian if $d^{+}(v)=d^{-}(v)$. The digraph $D$ is Eulerian if every vertex in $D$ is Eulerian. A digraph $D$ is $\Delta$-regular if $d^{+}(v)=d^{-}(v)=\Delta$ for all $v \in V(D)$. We say that $u$ is an out-neighbor (in-neighbor) of $v$ if $v u(u v)$ is an arc. We denote by $N^{+}(v)$ and $N^{-}(v)$ the set of out-neighbors and in-neighbors of $v$, respectively. The neighborhood of $v$, denoted by $N(v)$, is defined as $N(v)=N^{+}(v) \cup N^{-}(v)$. Every undirected graph $G$ determines a bidirected digraph $D(G)$ that is obtained from $G$ by replacing each edge with two oppositely directed edges joining the same pair of vertices. If $D$ is a digraph, we let $G(D)$ be the underlying undirected graph obtained from $D$ by "forgetting" all orientations. A digraph $D$ is said to be (weakly) connected if $G(D)$ is connected. The blocks of a digraph $D$ are the maximal subdigraphs $D^{\prime}$ of $D$ whose underlying undirected graph $G\left(D^{\prime}\right)$ is 2-connected. A cycle in a digraph $D$ is a cycle in $G(D)$ that does not use parallel edges. A directed cycle in $D$ is a subdigraph forming a directed closed walk in $D$ whose vertices are all distinct. A directed cycle consisting of exactly two vertices is called a digon.

The rest of the paper is organized as follows. In Section 2, we improve Brooks' bound for digraphs that have sufficiently large degrees. In Section 3, we consider the problem for arbitrary degrees.

\section{Strengthening Brooks' Theorem for large $\tilde{\Delta}$}

The main result in this section is the following theorem.

Theorem 2.1. There is an absolute constant $\Delta_{1}$ such that every digon-free digraph $D$ with $\tilde{\Delta}=\tilde{\Delta}(D) \geq \Delta_{1}$ has $\chi(D) \leq\left(1-e^{-13}\right) \tilde{\Delta}$.

The rest of this section is the proof of Theorem 2.1. The proof is a modification of an argument found in Molloy and Reed [11] for usual coloring of undirected graphs. We first note the following simple lemma.

Lemma 2.2. Let $D$ be a digraph with maximum out-degree $\Delta_{o}$, and suppose we have a partial proper coloring of $D$ with at most $\Delta_{o}+1-r$ colors. Suppose that for every vertex $v$ there are at least $r$ colors that appear on vertices in $N^{+}(v)$ at least twice. Then $D$ is $\Delta_{o}+1-r$-colorable.

Proof. The proof is easy - since many colors are repeated on the out-neighborhood of $v$, there are many colors that are not used on $N^{+}(v)$. Thus, one can greedily "extend" the partial coloring. 
Proof of Theorem 2.1. We assume that $\tilde{\Delta}$ is sufficiently large. We may also assume that $c_{1} \tilde{\Delta}<d^{+}(v)<c_{2} \tilde{\Delta}$ and $c_{1} \tilde{\Delta}<d^{-}(v)<c_{2} \tilde{\Delta}$ for each $v \in V(D)$, where $c_{1}=1-\frac{1}{3} e^{-11}$ and $c_{2}=1+\frac{1}{3} e^{-11}$. If not, we remove all the vertices $v$ not satisfying the above inequality and obtain a coloring for the remaining graph with $\left(1-e^{-13}\right) \tilde{\Delta}$ colors. Now, if a vertex does not satisfy the above condition either one of $d^{+}(v)$ or $d^{-}(v)$ is at most $c_{1} \tilde{\Delta}$ or one of $d^{+}(v)$ or $d^{-}(v)$ is at most $\frac{1}{c_{2}} \tilde{\Delta}$. Note that $1-e^{-13}>\max \left\{c_{1}, 1 / c_{2}\right\}$. This ensures that there is a color that either does not appear in the in-neighborhood or does not appear in the out-neighborhood of $v$, allowing us to complete the coloring.

The core of the proof is probabilistic. We color the vertices of $D$ randomly with $C$ colors, $C=\lfloor\tilde{\Delta} / 2\rfloor$. That is, for each vertex $v$ we assign $v$ a color from $\{1,2, \ldots, C\}$ uniformly at random. After the random coloring, we uncolor all the vertices that are in a monochromatic directed path of length at least 2. Clearly, this results in a proper partial coloring of $D$ since $D$ has no digons. For each vertex $v$, we are interested in the number of colors that are assigned to at least two out-neighbors of $v$ and are retained by at least two of these vertices. For analysis, it is better to define a slightly simpler random variable. Let $v \in V(D)$. For each color $i, 1 \leq i \leq C$, let $O_{i}$ be the set of out-neighbors of $v$ that have color $i$ assigned to them in the first phase. Let $X_{v}$ be the number of colors $i$ for which $\left|O_{i}\right| \geq 2$ and such that all vertices in $O_{i}$ retain their color after the uncoloring process.

For every vertex $v$, we let $A_{v}$ be the event that $X_{v}$ is less than $\frac{1}{2} e^{-11} \tilde{\Delta}+1$. We will show that with positive probability none of the events $A_{v}$ occur. Then Lemma 2.2 will imply that $\chi(D) \leq\left(c_{2}-\frac{1}{2} e^{-11}\right) \tilde{\Delta} \leq\left(1-e^{-13}\right) \tilde{\Delta}$, finishing the proof. We will use the symmetric version of the Lovász Local Lemma (see for example [2]). Note that the color assigned initially to a vertex $u$ can affect $X_{v}$ only if $u$ and $v$ are joined by a path of length at most 3. Thus, $A_{v}$ is mutually independent of all except at most $\left(2 c_{2} \tilde{\Delta}\right)+\left(2 c_{2} \tilde{\Delta}\right)^{2}+\left(2 c_{2} \tilde{\Delta}\right)^{3}+\left(2 c_{2} \tilde{\Delta}\right)^{4}+\left(2 c_{2} \tilde{\Delta}\right)^{5}+\left(2 c_{2} \tilde{\Delta}\right)^{6} \leq 100 \tilde{\Delta}^{6}$ other events $A_{w}$. Therefore, by the symmetric version of the Local Lemma, it suffices to show that for each event $A_{v}, 4 \cdot 100 \tilde{\Delta}^{6} \mathbb{P}\left[A_{v}\right]<1$. We will show that $\mathbb{P}\left[A_{v}\right]<\tilde{\Delta}^{-7}$. We do this by proving the following two lemmas.

Lemma 2.3. $\mathbb{E}\left[X_{v}\right] \geq e^{-11} \tilde{\Delta}-1$.

Proof. Let $X_{v}^{\prime}$ be the random variable denoting the number of colors that are assigned to exactly two out-neighbors of $v$ and are retained by both of these vertices. Clearly, $X_{v} \geq X_{v}^{\prime}$ and therefore it suffices to consider $\mathbb{E}\left[X_{v}^{\prime}\right]$.

Note that color $i$ will be counted by $X_{v}^{\prime}$ if two vertices $u, w \in N^{+}(v)$ are colored $i$ and no other vertex in $S=N(u) \cup N^{+}(v) \cup N(w)$ is assigned color $i$. This will give us a lower bound on $\mathbb{E}\left[X_{v}^{\prime}\right]$. There are $C$ choices for color $i$ and at least $\left(\begin{array}{c}c_{1} \tilde{\Delta} \\ 2\end{array}\right)$ choices for the set $\{u, w\}$. The probability that no vertex in $S$ other than $u$ and $w$ gets color $i$ is at least $\left(1-\frac{1}{C}\right)^{|S|} \geq\left(1-\frac{1}{C}\right)^{5 c_{2} \tilde{\Delta}}$. Therefore, by linearity of expectation, and using the fact that 
$(1-x)^{n} \geq e^{-n x-x}$, where $x=\theta(1 / n)$ and $n$ is sufficiently large, we can estimate:

$$
\begin{aligned}
\mathbb{E}\left[X_{v}^{\prime}\right] & \geq C\left(\begin{array}{c}
c_{1} \tilde{\Delta} \\
2
\end{array}\right)\left(\frac{1}{C}\right)^{2}\left(1-\frac{1}{C}\right)^{5 c_{2} \tilde{\Delta}} \\
& \geq c_{1}\left(c_{1} \tilde{\Delta}-1\right) \exp \left(-5 c_{2} \tilde{\Delta} / C-1 / C\right) \\
& \geq\left(c_{1}^{2} \tilde{\Delta}-c_{1}\right) \exp (-10.001) \\
& \geq \frac{\tilde{\Delta}}{e^{11}}-1,
\end{aligned}
$$

for $\tilde{\Delta}$ sufficiently large.

Lemma 2.4. $\mathbb{P}\left[\left|X_{v}-\mathbb{E}\left[X_{v}\right]\right|>\log \tilde{\Delta} \sqrt{\mathbb{E}\left[X_{v}\right]}\right]<\tilde{\Delta}^{-7}$.

Proof. Let $A T_{v}$ be the random variable counting the number of colors assigned to at least two out-neighbors of $v$, and $D e l_{v}$ the random variable that counts the number of colors assigned to at least two out-neighbors of $v$ but removed from at least one of them. Clearly, $X_{v}=A T_{v}-D e l_{v}$ and therefore it suffices to show that each of $A T_{v}$ and $D e l_{v}$ is sufficiently concentrated around its mean. We will show that for $t=\frac{1}{2} \log \tilde{\Delta} \sqrt{\mathbb{E}\left[X_{v}\right]}$ the following estimates hold:

Claim 1: $\mathbb{P}\left[\left|A T_{v}-\mathbb{E}\left[A T_{v}\right]\right|>t\right]<2 e^{-t^{2} /(8 \tilde{\Delta})}$.

Claim 2: $\mathbb{P}\left[\mid\right.$ Del $_{v}-\mathbb{E}\left[\right.$ Del $\left.\left._{v}\right] \mid>t\right]<4 e^{-t^{2} /(100 \tilde{\Delta})}$.

The two above inequalities yield that, for $\tilde{\Delta}$ sufficiently large,

$$
\begin{aligned}
\mathbb{P}\left[\left|X_{v}-\mathbb{E}\left[X_{v}\right]\right|>\log \tilde{\Delta} \sqrt{\mathbb{E}\left[X_{v}\right]}\right] & \leq 2 e^{-\frac{t^{2}}{8 \Delta}}+4 e^{-\frac{t^{2}}{100 \Delta}} \\
& \leq \tilde{\Delta}^{-\log \tilde{\Delta}} \\
& <\tilde{\Delta}^{-7}
\end{aligned}
$$

as we require. So, it remains to establish both claims.

To prove Claim 1, we use a version of Azuma's inequality found in [11], called the Simple Concentration Bound.

Theorem 2.5 (Simple Concentration Bound). Let $X$ be a random variable determined by $n$ independent trials $T_{1}, \ldots, T_{n}$, and satisfying the property that changing the outcome of any single trial can affect $X$ by at most $c$. Then

$$
\mathbb{P}[|X-\mathbb{E}[X]|>t] \leq 2 e^{-\frac{t^{2}}{2 c^{2} n}} .
$$

Note that $A T_{v}$ depends only on the colors assigned to the out-neighbors of $v$. Note that each random choice can affect $A T_{v}$ by at most 1 . Therefore, we can take $c=1$ in the Simple Concentration Bound for $X=A T_{v}$. Since the choice of random color assignments are made independently over the vertices and since $d^{+}(v) \leq c_{2} \tilde{\Delta}$, we immediately have the first claim.

For Claim 2, we use the following variant of Talagrand's Inequality (see [11]). 
Theorem 2.6 (Talagrand's Inequality). Let $X$ be a nonnegative random variable, not equal to 0 , that is determined by $n$ independent trials, $T_{1}, \ldots, T_{n}$ and satisfyies the following conditions for some $c, r>0$ :

1. Changing the outcome of any single trial can affect $X$ by at most $c$.

2. For any $s$, if $X \geq s$, there are at most rs trials whose exposure certifies that $X \geq s$.

Then for any $0 \leq \lambda \leq \mathbb{E}[X]$,

$$
\mathbb{P}[|X-\mathbb{E}[X]|>\lambda+60 c \sqrt{r \mathbb{E}[X]}] \leq 4 e^{-\frac{\lambda^{2}}{8 c^{2} r \mathbb{E}[X]}} .
$$

We apply Talagrand's inequality to the random variable $D e l_{v}$. Note that we can take $c=1$ since any single random color assignment can affect $D e l_{v}$ by at most 1 . Now, suppose that $D e l_{v} \geq s$. One can certify that $\operatorname{Del}_{v} \geq s$ by exposing, for each of the $s$ colors $i$, two random color assignments in $N^{+}(v)$ that certify that at least two vertices got color $i$, and exposing at most two other color assignments which show that at least one vertex colored $i$ lost its color. Therefore, $D e l_{v} \geq s$ can be certified by exposing $4 s$ random choices, and hence we may take $r=4$ in Talagrand's inequality. Note that $t=\frac{1}{2} \log \tilde{\Delta} \sqrt{\mathbb{E}\left[X_{v}\right]} \gg 60 c \sqrt{r \mathbb{E}\left[D e l_{v}\right]}$ since $\mathbb{E}\left[X_{v}\right] \geq \tilde{\Delta} / e^{11}-1$ and $\mathbb{E}\left[D e l_{v}\right] \leq c_{2} \tilde{\Delta}$. Now, taking $\lambda$ in Talagrand's inequality to be $\lambda=\frac{1}{2} t$, we obtain that $\mathbb{P}\left[\left|D e l_{v}-\mathbb{E}\left[D e l_{v}\right]\right|>t\right] \leq$ $\mathbb{P}\left[\left|\operatorname{Del}_{v}-\mathbb{E}\left[\operatorname{Del}_{v}\right]\right|>\lambda+60 c \sqrt{r \mathbb{E}[X]}\right]$. Therefore, provided that $\lambda \leq \mathbb{E}\left[\right.$ Del $\left._{v}\right]$, we have the confirmed Claim 2.

It is sufficient to show that $\mathbb{E}\left[\operatorname{Del}_{v}\right]=\Omega(\tilde{\Delta})$, since $\lambda=O(\log \tilde{\Delta} \sqrt{\tilde{\Delta}})$. The probability that exactly two vertices in $N^{+}(v)$ are assigned a particular color $x$ is at least $\frac{c_{1} \tilde{\Delta}^{2}}{2} C^{-2}(1-$ $1 / C)^{c_{2} \tilde{\Delta}} \approx 2 e^{-10}$, a constant. It remains to show that the probability that at least one of these vertices loses its color is also (at least) a constant. We use Janson's Inequality (see $[2]$ ). Let $u$ be one of the two vertices colored $x$. We only compute the probability that $u$ gets uncolored. We may assume that the other vertex colored $x$ is not a neighbor of $u$ since this will only increase the probability. We show that with large probability there exists a monochromatic directed path of length at least 2 starting at $u$. Let $\Omega=N^{+}(u) \cup N^{++}(u)$, where $N^{++}(u)$ is the second out-neighborhood of $u$. Each vertex in $\Omega$ is colored $x$ with probability $\frac{2}{\tilde{\Delta}}$. Enumerate all the directed paths of length 2 starting at $u$ and let $P_{i}$ be the $i^{\text {th }}$ path. Clearly, there are at least $\left(c_{1} \tilde{\Delta}\right)^{2}$ such paths $P_{i}$. Let $A_{i}$ be the set of vertices of $P_{i}$, and denote by $B_{i}$ the event that all vertices in $A_{i}$ receive the same color. Then, clearly $\mathbb{P}\left[B_{i}\right]=\frac{1}{((\tilde{\Delta} / 2])^{2}} \geq \frac{4}{\tilde{\Delta}^{2}}$. Then, $\mu=\sum \mathbb{P}\left[B_{i}\right] \geq \frac{4}{\tilde{\Delta}^{2}} \cdot\left(c_{1} \tilde{\Delta}\right)^{2}=4 c_{1}^{2}$. Now, if $\delta=\sum_{i, j: A_{i} \cap A_{j} \neq \emptyset} \mathbb{P}\left[B_{i} \cap B_{j}\right]$ in Janson's Inequality satisfies $\delta<\mu$, then applying Janson's Inequality, with the sets $A_{i}$ and events $B_{i}$, we obtain that the probability that none of the events $B_{i}$ occur is at most $e^{-1}$, and hence the probability that $u$ does not retain its color is at least $1-e^{-1}$, as required. Now, assume that $\delta \geq \mu$. The following gives an 
upper bound on $\delta$ :

$$
\begin{aligned}
\delta & =\sum_{i, j: A_{i} \cap A_{j} \neq \emptyset} \mathbb{P}\left[B_{i} \cap B_{j}\right]=\sum_{i, j: A_{i} \cap A_{j} \neq \emptyset} \frac{1}{(\lfloor\tilde{\Delta} / 2\rfloor)^{3}} \\
& \leq\left(c_{2} \tilde{\Delta}\right)^{2} \cdot 2 c_{2} \tilde{\Delta} \cdot \frac{8}{(\tilde{\Delta}-2)^{3}}<32
\end{aligned}
$$

for $\tilde{\Delta} \geq 100$. Now, we apply Extended Janson's Inequality (again see [2]). This inequality now implies that the probability that none of the events $B_{i}$ occur is at most $e^{-c_{1}^{2} / 4}$, a constant. Therefore, by linearity of expectation $\mathbb{E}\left[D e l_{v}\right]=\Omega(\tilde{\Delta})$.

Clearly, since $\mathbb{E}\left[X_{v}\right] \leq c_{2} \tilde{\Delta}$, Lemmas 2.3 and 2.4 imply that $\mathbb{P}\left[A_{v}\right]<\tilde{\Delta}^{-7}$. This completes the proof of Theorem 2.1.

\section{Brooks' Theorem for small $\tilde{\Delta}$}

The bound in Theorem 2.1 is only useful for large $\tilde{\Delta}$. Rough estimates suggest that $\tilde{\Delta}$ needs to be at least in the order of $10^{10}$. The above approach is unlikely to improve this bound significantly with a more detailed analysis. In this section, we improve Brooks' Theorem for all values of $\tilde{\Delta}$. We achieve this by using a result on list colorings found in [6]. List coloring of digraphs is defined analogously to list coloring of undirected graphs. A precise definition is given below.

Let $\mathcal{C}$ be a finite set of colors. Given a digraph $D$, let $L: v \mapsto L(v) \subseteq \mathcal{C}$ be a listassignment for $D$, which assigns to each vertex $v \in V(D)$ a set of colors. The set $L(v)$ is called the list (or the set of admissible colors) for $v$. We say $D$ is $L$-colorable if there is an $L$-coloring of $D$, i.e., each vertex $v$ is assigned a color from $L(v)$ such that every color class induces an acyclic subdigraph in $D$. $D$ is said to be $k$-choosable if $D$ is $L$-colorable for every list-assignment $L$ with $|L(v)| \geq k$ for each $v \in V(D)$. We denote by $\chi_{l}(D)$ the smallest integer $k$ for which $D$ is $k$-choosable.

The result characterizes the structure of non $L$-colorable digraphs whose list sizes are one less than given by the directed version of Brooks' theorem.

Theorem $3.1([6])$. Let $D$ be a connected digraph, and $L$ an assignment of colors to the vertices of $D$ such that $|L(v)| \geq d^{+}(v)$ if $d^{+}(v)=d^{-}(v)$ and $|L(v)| \geq \min \left\{d^{+}(v), d^{-}(v)\right\}+1$ otherwise. Suppose that $D$ is not L-colorable. Then $D$ is Eulerian, $|L(v)|=d^{+}(v)$ for each $v \in V(D)$, and every block of $D$ is one of the following:

(a) a directed cycle (possibly a digon),

(b) an odd bidirected cycle, or

(c) a bidirected complete digraph.

Now, we can state the next result of this section. 
Lemma 3.2. Let $D$ be a connected digraph without digons, and let $\tilde{\Delta}=\tilde{\Delta}(D)$. If $\tilde{\Delta}>1$, then $\chi_{l}(D) \leq\lceil\tilde{\Delta}\rceil$.

Proof. We apply Theorem 3.1 with all lists $L(v), v \in V(D)$ having cardinality $\lceil\tilde{\Delta}\rceil$. It is clear that the conditions of Theorem 3.1 are satisfied for every Eulerian vertex $v$. It is easy to verify that the conditions are also satisfied for non-Eulerian vertices. Now, if $D$ is not $L$-colorable, then by Theorem 3.1, $D$ is Eulerian and $d^{+}(v)=\lceil\tilde{\Delta}\rceil$ for every vertex $v$. This implies that $D$ is $\lceil\tilde{\Delta}\rceil$-regular. Now, the conclusion of Theorem 3.1 implies that $D$ consists of a single block of type (a), (b) or (c). This means that either $D$ is a directed cycle (and hence $\tilde{\Delta}=1$ ), or $D$ contains a digon, a contradiction. This completes the proof.

We can now prove the main result of this section, which improves Brooks' bound for all digraphs without digons.

Theorem 3.3. Let $D$ be a connected digraph without digons, and let $\tilde{\Delta}=\tilde{\Delta}(D)$. If $\tilde{\Delta}>1$, then $\chi(D) \leq \alpha(\tilde{\Delta}+1)$ for some absolute constant $\alpha<1$.

Proof. We define $\alpha=\max \left\{\frac{\Delta_{1}}{\Delta_{1}+1}, 1-e^{-13}\right\}$, where $\Delta_{1}$ is the constant in the statement of Theorem 2.1. Now, if $\tilde{\Delta}<\Delta_{1}$ then by Lemma 3.2, it follows that $\chi(D) \leq\lceil\tilde{\Delta}\rceil \leq \alpha(\tilde{\Delta}+1)$. If $\tilde{\Delta} \geq \Delta_{1}$, then by Theorem 2.1 we obtain that $\chi(D) \leq\left(1-e^{-13}\right) \tilde{\tilde{\Delta}} \leq \alpha(\tilde{\Delta}+1)$, as required.

An interesting question to consider is the tightness of the bound of Lemma 3.2. It is easy to see that the bound is tight for $[\tilde{\Delta}\rceil=2$ by considering, for example, a directed cycle with an additional chord or a digraph consisting of two directed triangles sharing a common vertex. The graph in Figure 1 shows that the bound is also tight for $\lceil\tilde{\Delta}\rceil=3$. It is easy to verify that, up to symmetry, the coloring outlined in the figure is the unique 2-coloring. Now, adding an additional vertex, whose three out-neighbors are the vertices of the middle triangle and the three in-neighbors are the remaining vertices, we obtain a 3 -regular digraph where three colors are required to complete the coloring.

Another example of a digon-free 3-regular digraph on 7 vertices requiring three colors is the following. Take the Fano Plane and label its points by $1,2, \ldots, 7$. For every line of the Fano plane containing points $a, b, c$, take a directed cycle through $a, b, c$ (with either orientation). There is a unique directed 3-cycle through any two vertices because every two points line in exactly one line. This shows that the Fano plane digraphs are not isomorphic to the digraph from the previous paragraph. Finally, it is easy to verify that the resulting digraph needs three colors for coloring.

Note that the digraphs in the above examples are 3-regular tournaments on 7 vertices. It is not hard to check that every tournament on 9 vertices has $\lceil\tilde{\Delta}\rceil=4$, and yet is 3 -colorable. In general, we pose the following problem.

Question 3.4. What is the smallest integer $\Delta_{0}$ such that every digraph $D$ without digons with $\lceil\tilde{\Delta}(D)\rceil=\Delta_{0}$ satisfies $\chi(D) \leq \Delta_{0}-1$ ? 


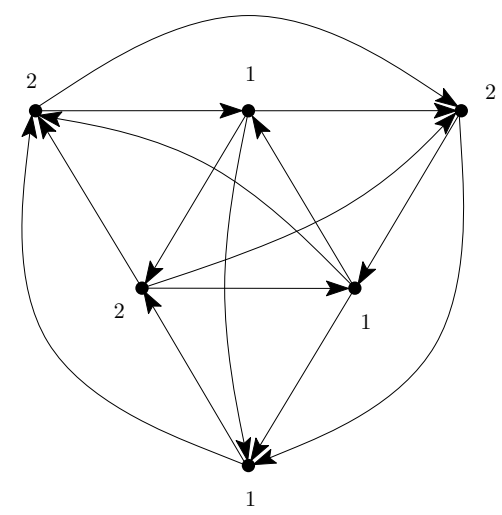

Figure 1: Constructing a 3-regular digraph $D$ with $\chi(D)=3$.

Note that this is a weak version of Conjecture 1.5. By Theorem 2.1, $\Delta_{0}$ exists. However, we believe that $\Delta_{0}$ is small, possibly equal to 4 . The following proposition shows that the above holds for every $\lceil\tilde{\Delta}\rceil \geq \Delta_{0}$.

Proposition 3.5. Let $\Delta_{0}$ be defined as in Question 3.4. Then every digon-free digraph $D$ with $\lceil\tilde{\Delta}(D)\rceil \geq \Delta_{0}$ satisfies $\chi(D) \leq\lceil\tilde{\Delta}(D)\rceil-1$.

Proof. The proof is by induction on $\lceil\tilde{\Delta}\rceil$. If $\lceil\tilde{\Delta}\rceil=\Delta_{0}$ this holds by the definition of $\Delta_{0}$. Otherwise, let $U$ be a maximal acyclic subset of $D$. Then $\lceil\tilde{\Delta}(D-U)\rceil \leq\lceil\tilde{\Delta}(D)\rceil-1$ for otherwise $U$ is not maximal. Since we can color $U$ by a single color, we can apply the induction hypothesis to complete the proof.

As a corollary we get:

Corollary 3.6. There exists a positive constant $\alpha<1$ such that for every digon-free digraph $D$ with $\lceil\tilde{\Delta}(D)\rceil \geq \Delta_{0}, \chi(D) \leq \alpha\lceil\tilde{\Delta}\rceil$.

Proof. Let $\alpha=\max \left\{\frac{\left\lceil\Delta_{1}\right\rceil}{\left\lceil\Delta_{1}\right\rceil+1}, 1-e^{-13}\right\}$, where $\Delta_{1}$ is the constant in the statement of Theorem 2.1. Now, applying Theorem 2.1 or Proposition 3.5 gives the result.

\section{References}

[1] R. Aharoni, E. Berger, O. Kfir, Acyclic systems of representatives and acyclic colorings of digraphs, Journal of Graph Theory 59 (2008) 177-189.

[2] N. Alon, J. Spencer, The Probabilistic Method, Wiley, 1992.

[3] J. Bang-Jensen, G. Gutin, Digraphs. Theory, Algorithms and Applications, Springer, 2001.

[4] D. Bokal, G. Fijavž, M. Juvan, P. M. Kayll, B. Mohar, The circular chromatic number of a digraph, Journal of Graph Theory 46 (2004) 227-240. 
[5] P. Erdős, J. Gimbel, D. Kratsch, Some extremal results in cochromatic and dichromatic theory, Journal of Graph Theory 15 (1991) 579-585.

[6] A. Harutyunyan, B. Mohar, Gallai's theorem for list coloring of digraphs, SIAM Journal on Discrete Mathematics 25 (2011) 170-180.

[7] A. Johansson, Asymptotic choice number for triangle free graphs, DIMACS Technical Report (1996) 91-95.

[8] C. McDiarmid, B. Mohar, private communication, 2002.

[9] B. Mohar, Circular colorings of edge-weighted graphs, Journal of Graph Theory 43 (2003) 107-116.

[10] B. Mohar, Eigenvalues and colorings of digraphs, Linear Algebra and its Applications 432 (2010) 2273-2277.

[11] M. Molloy, B. Reed, Graph Colouring and the Probabilistic Method, Springer, 2002.

[12] V. Neumann-Lara, The dichromatic number of a digraph, Journal of Combinatorial Theory, Series B 33 (1982) 265-270.

[13] V. Neumann-Lara, Vertex colourings in digraphs - some problems, Seminar notes (communicated by A. Bondy and S. Thomassé), Waterloo, Canada, July 8, 1985.

[14] B. Reed, $\omega, \Delta$, and $\chi$, Journal of Graph Theory 27 (1998) 177-212. 